\title{
Frame analysis of psychotherapists' perspectives on the development of psychological disorders
}

\author{
Eve Riachi ${ }^{1}$ (D) Juha Holma ${ }^{1}$ (D) Aarno Laitila ${ }^{1}$ (D)
}

Accepted: 9 August 2021

(C) The Author(s) 2021

\begin{abstract}
The development of psychological disorders has been explained by several psychological theories and remains under debate. Psychotherapists, however, have insights into the emergence and development of psychological disorders that stem from both theory and practice. The constantly evolving field of psychotherapy prompts reconsideration, specifically when psychotherapists' views on the development of disorders impacts their treatment approach. In addition, theoretical orientation and years of clinical experience, while known to influence psychotherapists' viewpoints also merit further study. Applying Erving Goffman's frame analysis, semi structured interviews with psychotherapists were conducted to determine their perspectives on the emergence of mental disorders. Biological, environmental, and psychological factors were mentioned both separately and in combination by the therapists. These factors, or frames, were then analyzed in relation to the therapists' therapeutic approaches and length of clinical experience. The analysis showed that the frames employed by the therapists were influenced by several therapeutic orientations. Moreover, therapists with more than ten years of clinical experience mentioned a combination of factors slightly more often than single factors alone. In sum, the findings showed that therapists perceived the development of mental disorders as highly complex and as an outcome of multiple factors. War was also mentioned as an environmental factor in the development of mental disorders. As this finding was clearly a country-specific environmental factor, future studies should explore the possible role of country-specific environmental factors in different countries.
\end{abstract}

Keywords Psychotherapists · Perspective of psychotherapists · Development of psychological disorders · Psychological disorders · Frame analysis · Qualitative research

\section{Introduction}

The increasing importance accorded to mental health has led to increased interest in the development of psychotherapy (Ronnestad and Skovholt, 2012). Psychotherapy is constantly evolving in an effort to provide better treatment outcomes. The fifth edition of the Diagnostic and Statistical Manual of Mental Disorders (5th ed.; DSM-5) is the latest of many updates and revisions in the psychotherapy field (American Psychiatric Association, 2013). In linking psychological theories to practice, psychotherapists play a central role in this developmental process. They integrate their knowledge of psychotherapeutic approaches with their own understanding

Eve Riachi

everiachi@gmail.com

1 Department of Psychology, University of Jyväskylä, Jyväskylä, Finland and experience to guide them in the treatment of disorders (Ahn et al., 2009). Psychotherapists greatly influence the progress of psychotherapy and have a substantial impact on their clients. Therapists also gain applied knowledge of psychotherapy through their clinical practice. Exploring the information psychotherapists acquire is critical in seeking to improve psychotherapy.

A fundamental component of psychotherapy is the therapist's understanding of the development of mental disorders, as this plays an important role in the therapeutic method they adopt in their clinical practice (Ahn et al., 2009). Psychotherapists' views on the development of disorders have been addressed in a few studies. Mental health clinicians have associated the development of disorders with a number of biological and psychosocial factors (Ahn et al., 2009). Miresco \& Kirmayer (2006) found that psychologists and psychiatrists separated the development of disorders into psychological and neurobiological. Moreover, some disorders were viewed as caused either biologically or environmentally and psychologically (Ahn et al., 2009). In the biologically 
caused disorders, genetics, and hereditary factors as well as biochemical imbalances in the brain were emphasized. Psychological and environmental factors in turn were linked to interpersonal relationships and life experiences. The more a disorder was judged to be caused biologically, the less the emphasis placed on psychological and environmental causes. In another study, mental health experts disagreed with the idea that mental disorders have a clear origin (Ahn et al., 2006). While some research has been conducted on therapists' viewpoints on the emergence of disorders, the constantly changing nature of psychotherapy calls for regular reconsideration on this topic (Ronnestad and Skovholt, 2012). Moreover, the current perspectives of psychotherapists could be further investigated to gain a more in-depth understanding of the development of therapists' professional opinions.

Psychotherapists' perspectives on the emergence of disorders are shaped by several factors, including psychotherapy training, theoretical orientation, professional experience, and personal experience (Skovholt, 2012). Psychotherapists seek to repair or manage mental disorders by applying specific psychotherapeutic approaches or psychological theories (Cave, 1999). The psychodynamic approach emphasizes the struggle between our desires and society. This struggle functions as a precondition, internalized during the developmental years, and is thought to be repressed in the unconscious. The approach focuses mainly on the unconscious and its effects on behavior (Leitan and Murray, 2014). It also discusses object relation theory and a child's attachment to primary caregivers (Cave, 1999). The mental representation of the primary caregiver as well as early childhood experiences influence how individuals relate to the world and others.

The behavioral approach views disorders, especially maladaptive behaviors, through the process of learning (Cave, 1999). In this approach, the environment plays an important role in shaping behavior; individuals learn, based on their experiences, how to behave in certain situations (Leitan and Murray, 2014). The cognitive approach, in turn, suggests that dysfunctional thoughts or cognitions are at the root of problematic behavior (Cave, 1999). In this approach, the human mind is viewed as a processor of experiences and a generator of thoughts (Leitan and Murray, 2014).

The humanistic approach relates mental disorders to an individual's deviation from their true self and as an impediment to self-development (Cave, 1999). The therapeutic relationship between the therapist and the client acts as the foundation for therapy. Eclecticism and integrative therapies focus on different psychotherapy techniques and aim to improve treatment prospects (Norcross and Goldfried, 2005). Eclecticism selects techniques from several therapies based on the effectiveness of these techniques with respect to the client's needs. Integrative theories, in turn, combine common research-based factors from several therapies, to formulate a psychotherapeutic approach that is suited to the client. There are, however, a few similarities between these therapeutic orientations. For example, while the eclectic and integrative theoretical approaches are based on different therapeutic approaches, both are modified by the client's therapeutic needs (Norcross and Goldfried, 2005). Moreover, the personal interpretation of experiences, and the importance of the meanings derived from these experiences, is an element common to most psychotherapeutic approaches.

With respect to professional experience, studies have shown that therapists' approaches are affected by the length of their clinical experience (Dawson, 2018). As they gain experience, therapists also develop and customize their therapeutic approach. Therapists considered as experts tend to have at least 10 years of working experience, and the more experienced therapists are, the more secure they feel in their clinical decision making and case conceptualization. Length of clinical experience is associated with therapists' sense of competence and tolerance of ambiguity. Experienced psychotherapists comprehend psychological processes in more complex ways (Ronnestad and Skovholt, 2012).

Research on the development of disorders is an integral part of psychology, and psychotherapists possess important information on the topic. Consequently, a more comprehensive understanding of psychotherapists' views on the emergence of disorders is needed to further develop psychotherapy. Theoretical orientation and professional experience are two major influences known to impact psychotherapists' perspectives and treatment approach. However, their impact on the way therapists currently view the development of mental disorders is not known. To contribute information on this issue, this study had three aims: first, to identify the different perspectives of psychotherapists on the development of disorders; second, to analyze therapists' explanations of the development of disorders in relation to their therapeutic approaches; and third, to explore the association between therapists' perspectives on the development of disorders and their years of clinical experience as a therapist.

\section{Method}

\section{Participants and Procedure}

Participants were Lebanese psychotherapists selected from various online platforms that allow individuals seeking therapy to connect with therapists. Around sixty Lebanese psychotherapists with different therapeutic backgrounds were contacted. The main inclusion criterion was working as a psychotherapist. Geographical convenience and a good command of the English language were also considered. It was important for therapists to be able to express their opinions in English. Having studied psychology or received training in English was considered an advantage. 
Sixteen Lebanese psychotherapists agreed to participate in the study, ten of them were females and six were males. Nine of the participants had a master's degree in clinical psychology, five had a doctorate in clinical psychology, and two had a bachelor's degree in psychology and specialized training in psychotherapy. They mainly reported applying psychodynamic, humanistic, trauma, cognitive and behavioral (CBT), eclectic, and integrative approaches. Several participants reported identifying with more than one approach. The psychotherapists either had training in the approach they used in therapy or had received supervised hours of therapy training as part of their degree studies. Participants' length of experience working as a psychotherapist ranged between three and thirty-eight years. Some of the therapists worked in psychiatric units, others in public organizations focusing on providing affordable mental health services, and most had their own private practice.

Participants were contacted by phone or email and invited to participate in the study. Information was given on the purpose of the study and the study procedure. Participants were informed that their perspectives on the development of psychological disorders and the role of loss of a sense of control would be studied. They were further informed that the study was qualitative, and that the data would be gathered during an interview conducted in English. Although Arabic is the official national language in Lebanon, English is a medium in education. The aim of interviewing the participants in English was to obtain data on their choice of words when expressing their opinions, and this was deemed best achieved by minimizing the use of translation.

Participants were also told that the interview would be audio-recorded. After participants had given their verbal consent, a date for their face-to-face interview was arranged. Before starting the interview, the research was described in more detail and participants could ask questions. Participants were then asked to give their written consent, after which the interview was conducted.

The interviews were semi-structured. Thirteen questions were asked in the interview, five of which were on demographics. The interview questions are shown in Appendix 1. All interviews were in English except for one, which was held mostly in Arabic and subsequently translated into English. Some therapists also used Arabic to explain specific thoughts, and these utterances were also translated into English. All the recorded interviews were transcribed with the exception of one that was given in writing by a participant who was unwilling to be audio-recorded. The preliminary work of data collection and coding was done by the first author. The data were then examined and analyzed in consultation with the other authors. Data analysis sessions were conducted once a month.

Participants were informed that their participation was voluntary and that their personal information would remain confidential throughout the research process. To guarantee informed consent and to protect the rights of participants, they were provided with a copy of the privacy agreement, which contained a detailed description of the research. Participants were also provided with a copy of their written consent form signed by both the participant and the researcher. To ensure participant anonymity, participants' names were not included in any of the saved data.

\section{Analysis}

Frame analysis is a research methodology developed by Erving Goffman in which data are grouped into frames (Goffman, 1974). Frames represent different interpretations of reality. Frames describe people's views on a certain topic based on their understanding and knowledge of that topic. Understanding is built on categorizations of social situations. Frame analysis is utilized in several research fields such as sociology, politics, media, and cultural studies as well as in psychology (Shaw, 2013).

In this study, frame analysis was employed to examine the different views of psychotherapists on the development of psychological disorders. This method of analysis was chosen to gain understanding of therapists' views on disorders as an outcome of integration between their chosen therapeutic approach, training, and professional experience. Atlas.ti software was used in analyzing and grouping the data. Each interview was analyzed based on the explanations the interviewees gave for the emergence of psychological disorders. Frame analysis was used to explore and categorize these different perspectives. Each explanation represented a specific idea discussed by the therapist. Frames were created by grouping similar explanations. These frames were then examined in relation to the therapists' therapeutic orientation and length of experience as a therapist. The therapeutic orientation of the therapists and the number of years they had worked as a psychotherapist were collected in the interviews as part of the demographic data.

The analysis and interpretation of the results was done by all three authors. The first author has no specific psychotherapeutic training and completed university studies in clinical psychology with special focus on several therapeutic approaches. The other two authors have psychotherapy training in systemic therapy and follow an integrative approach.

\section{Results}

The first research question addressed the psychotherapists' views on the development of disorders. The frame analysis showed that the emergence of psychological disorders, as defined in this study, could be grouped under two main categories or frames: single frames and combinations of frames. Single frames comprised individual factors discussed by the 
therapists in isolation, that is, as unconnected to other factors. Combinations of frames comprised explanations in which the main focus was on two or more factors. The single factors were labeled biological, environmental, and psychological. Combinations of factors contained two frames: a combination of factors from the biological and environmental frames and a combination of factors from several frames.

Single factors were mentioned by the therapists slightly more often than combinations of factors from different frames. Moreover, of the single frames, factors in the environmental frame were the most frequently mentioned. Combinations of factors from both the biological and environmental frames were the second most often mentioned. The frames and their frequencies are shown in Table 1.

The environmental frame comprised five subgroups: childhood and upbringing, trauma, loss of sense of control, unspecified environmental factors, and cultural factors. The most often mentioned environmental factors were childhood and upbringing. The second most mentioned was trauma, followed by loss of a sense of control. The five subgroups and their frequencies are also shown in Table 1.

\section{Single Frames}

\section{Biological Frame}

The biological frame comprised therapists' explanations that suggested biological factors as a distinct component in the development of disorders. In these explanations, the role of biology in mental disorders, and hence the identification of biological factors, was seen as an important component on its own, and unrelated to any other factors.

The psychotherapists discussed biological factors using terms such as genetics, genetic predispositions, family history

Table 1 Frames describing psychotherapists' views on the development of disorders and their frequencies

\begin{tabular}{ll}
\hline Frames & Frequency \\
\hline Single Frames & $\mathbf{3 5}$ \\
1. Biological Frame & 2 \\
2. Psychological Frame & 5 \\
3. Environmental Frame & 28 \\
a. Childhood and Upbringing & 14 \\
b. Trauma & 6 \\
c. Loss of Sense of Control & 5 \\
d. Cultural Factors & 1 \\
e. Unspecified Environmental Factors & 2 \\
Combination of Frames & $\mathbf{2 3}$ \\
1. Combination of Biological and Environmental Frames & 16 \\
2. Combination of Several Frames & 7 \\
\hline
\end{tabular}

and genetic background, individual vulnerabilities, temperament, and biochemical imbalances.

Interview 12: ... if you want me to simplify things, we are born with certain weaknesses, so some people are more prone to developing certain psychological disorders than others. ... there is something genetic, there is something built in with certain disorders.

\section{Psychological Frame}

The psychological frame included explanations focusing on the role of individual psychological processes in the emergence of mental disorders. Unrelated to other factors, the psychological frame included identity, self-image, and awareness. Self-knowledge and awareness of one's mental health was seen as an essential component of overall well-being.

Interview 3: She gave me an example about her mom, telling her she has to lose some weight but she tells her mom I am fine with who I am in my body. In reality she doesn't like her body, she doesn't like herself. So her identity is also confused.

Interview 8: ...it is not knowing what is going on with you. There is no awareness and it gets to the point of suddenly having a disorder because you have never actually worked on fixing anything so it doesn't reach that point.

\section{Environmental Frame}

The environmental frame included explanations that highlight the role of the external environment in the development of disorders. Environmental factors are components of an individual's surroundings that influence the individual. Many environmental factors were mentioned by the therapists irrespective of whether or not they believed the environment alone plays a major role in the development of disorders. These factors were exposure to trauma, family environment and upbringing, issues with love and attachment, life experiences, relocation or changes in an individual's milieu, wars and epidemics, weather, bullying, relational problems, financial problems, and level of education.

In this frame, environmental factors were discussed separately from other factors that could contribute to the emergence of disorders. While they had a clear role in the development of disorders, they differed in the explanations they offered and were therefore sorted into different subgroups. The subgroups were childhood and upbringing, trauma, loss of sense of control, unspecified environmental factors, and cultural factors. 
Childhood and Upbringing Subgroup This subgroup contained the most frequently mentioned environmental factors, including family problems as well as parent-child relationships, especially communication problems, attachment issues, and parental attunement to the child's needs. Unmet childhood needs were also discussed in connection with cognition, and the effects of unmet needs on the child's perception. Abuse and neglect were part of this subgroup, as were childhood and teenage experiences. Abuse and neglect were integrated in this subgroup, as their co-occurrence in childhood was specifically referred to by the therapists. Moreover, negative childhood events that affect self-esteem and contribute to the creation of negative cognitions about the self were also discussed in this subgroup.

Interview 3: ... abuse plays a major role, and by abuse I also have in mind neglect. Also communication problems play a major role especially between parents and children. ... attachment problems as well are included in communication problems between parents and children. This is all during childhood. If I am going to consider the systemic approach, when a family member has a certain difficulty or problem, usually the whole system gets affected and with time this creates issues. ... things happen in an individual's childhood, with time negative events create in the individual negative self-beliefs and this affects their self-esteem. Examples of negative cognitions are "I am weak" or "I am not lovable" or "People are bad".

Interview 2: I work with needs, what were the needs of the child that didn't get met in childhood and how did that impact their cognitions and the way they view the world today.

Trauma Subgroup Trauma-related factors formed the second most frequently mentioned subgroup and were not connected to other factors. This subgroup consisted of explanations focusing on the role of trauma, including severe trauma and its effects in shaping an individual's feelings, thoughts, and behaviors. Negative experiences and their ability to create negative cognitions were also discussed in addition to relational and emotional hurt.

Therapists who discussed trauma as a main factor in the emergence of disorders defined trauma as negative experiences or as life situations in general. One definition focused on the neurological component of trauma and the formation of trauma based on the abnormal processing of certain life situations. Other definitions of trauma included active abuse, whether verbal, emotional or physical. The last definition discussed the unmet needs of an organism preceding birth. The supply of oxygen and safety as well as the absence of other factors such as stress hormones, sound pollution or smoke were given as examples in this definition.

The trauma subgroup shared some features with the childhood and upbringing subgroup. Negative experiences were discussed in both subgroups but with different underlying factors: in the trauma subgroup, negative experiences were discussed in relation to trauma but without any reference to childhood and upbringing. The cognitive component was also mentioned in both groups, but the reason given for the development of negative cognition was different. In the trauma subgroup, negative cognitions were seen as an outcome of trauma, whereas in the childhood and upbringing subgroup negative cognitions were seen as a result of unmet childhood needs. Both trauma and unmet needs were considered to affect an individual and lead to the development of negative cognitions.

Interview 6: There is a lot of pathology that develops without any predisposition. We have individuals with no predisposition to any mental illness but they undergo severe enough, bad enough traumas that they develop symptoms including psychosis.

Interview 4: I believe that certain traumas in your life which are life situations, incidents, or environments shape you into being who you are today, what you believe about yourself, what you believe about the world, how you feel and how you behave. ... for anxious people for example, there is this certain underlying schema or underlying belief that says "I am in danger" or "I am not safe". For depressed people it is "I am helpless" or "I am powerless".

Loss of Sense of Control Subgroup Loss of a sense of control was associated with an inability to deal with or adapt to a certain situation and was discussed as an independent factor in the development of disorders. This subgroup was categorized under the environmental frame rather than the psychological frame, as the therapists saw the environment as the origin of the loss of a sense of control. Although loss of a sense of control is considered a psychological process, the environment, or events in the environment are responsible for the feelings of loss of a sense of control and the inability to deal with or adapt to certain situations. These therapists discussed the feeling of being stuck and failure to change one's present circumstances. The term sense of control refers to an individual's perception of their ability to control or gain control over their life (Shapiro, 1994). Loss of a sense of control is thus a perceived inability to gain control. A few explanations were offered for the loss of a sense of control. 
Interview 12: ... there is something built in with certain disorders, maybe some others it is not genetic, it is purely environmental and the person couldn't develop and deal with that and things happen.

Cultural Factors Subgroup In the cultural factors subgroup, culture was identified as an independent component in the emergence of disorders. The influence of culture on mental health and the ability to seek help and treatment were discussed.

Interview 8: ...still I think the culture plays a huge role in covering up and belittling anything that has to do with mental health so that doesn't help people.

Unspecified Environmental Factors Subgroup This subgroup contained discussion on the major role played by the environment in the development of disorders but without focusing on any specific environmental factors. For the psychotherapists in this subgroup, the environment comprises a multiplicity of variables, including childhood and upbringing, trauma, contextual factors such as an individual's surroundings and changes in these, relocation, financial and social problems, relational problems, and level of education.

Interview 2: ...especially for things like personality disorders, environment really does have a considerable impact.

Interview 10: ...there is obviously a genetic factor in many cases and in other cases it is environmental factors ...

\section{Combinations of Frames}

\section{Combination of Biological and Environmental Frames}

Some of the psychotherapists discussed the combination of both biological and environmental factors in the emergence of disorders. Thus, this frame comprised two other frames, the biological and the environmental. The biological frame contained discussion about genetics, genetic predisposition, being born with certain predispositions, individual vulnerability, and biochemical imbalances. The term nature versus nurture was also mentioned, with nature referring to biological factors and nurture to environmental factors. The environmental frame, in turn, referred to such factors as the environment, exposure to trauma, external stressors, and an unhealthy social or relational dynamic.
In the talk assigned to this frame, the focus was on both factors equally. These psychotherapists discussed biological and environmental factors simultaneously in their descriptions of the development of disorders.

Interview 4: I think disorders develop because we are born with certain predisposition to certain disorders and then there is the factor of environment that plays a huge role.

Interview 7: Basically there is always the nurture and nature component... any exposure to trauma will cause any predisposed psychological disorder to be activated.

\section{Combination of Several Frames}

This frame comprised explanations by therapists who saw mental disorders as an outcome of multiple factors with parallel emphasis. The main factors discussed included environmental factors, perception of the environment, cognition, biopsychosocial factors, interpersonal factors, physical health, genetics, genetic resilience, predisposition to using drugs and alcohol, temperament, family, attachment issues, trauma and the timing, severity, and repetition of a trauma. The combination of the environmental, psychological, and biological frames is exemplified below.

Interview 6: We have seen that the timing of the trauma, the kind of trauma, the intensity, the repetition and any other factors including genetic resilience or genetic predisposition to develop pathology. All this stuff does affect the outcome in terms of what kind of symptoms, how severe, which disorder develops.

Interview 11: Several things, several components are involved in the development of a psychological disorder. There is the genetic part that can play an important role, the environment that plays an important role, the personality, the temperament of the person, life events, exposure, the way of thinking, the learning of the way of thinking and of managing thinking and emotions.

Interview 15: We were all born with different biology, with different abilities and different needs and emotional regulatory skills; and mainly our environment, our caregivers roles, our chronic interactions with our parents or primary caregivers plays a big part in our symptoms.

\section{Frames and Psychotherapeutic Approaches}

The second research question examined psychotherapists' views on the development of disorders in relation to their 
therapeutic approaches. The psychotherapists who participated in this study were asked about their therapeutic orientation. During the interview, several psychotherapeutic approaches were listed verbally in a multiple-choice format from which therapists could choose the ones that most applied to them. The therapists were also free to add a therapeutic orientation that was not included in the interview question. The therapists selected the following main therapies: psychodynamic therapy, cognitive-behavioral therapy, humanistic therapy, trauma therapy, integrative therapy, and eclectic therapy. Psychoanalysis was combined with psychodynamic approaches, eye movement desensitization and reprocessing (EMDR) was combined with trauma therapy, and dialectical behavioral therapy or DBT was combined with cognitive behavioral therapy. These combinations of approaches were based on the similarities between them. Such combinations also facilitate analysis and aid in exploring the main psychotherapeutic approaches and the different ways in which each approach explains the development of disorders. The psychotherapists identified with one or more psychotherapeutic approaches. Their responses are shown in Table 2.

The frames, discussed previously, were then analyzed in relation to the main therapeutic approaches identified by the therapists. This permitted assessment of the resemblance between the therapists' perceptions on the development of disorders and their psychotherapeutic approaches. The number of participants in each therapy group is unequally distributed, and thus a reliable comparison between therapeutic approaches is not possible. Normalizing the data provides an equal average for each therapy group which then permits comparison. Table 3 presents the normalized version of the data as well as the relative frequencies in percentages.

The frames that were mentioned across all therapeutic approaches were the environmental frame, combination of the environmental and biological frames, and the combination of several frames. The cognitive behavioral therapists, humanistic therapists, and trauma therapists focused mostly on the combination of environmental and biological frames. The eclectic therapists, integrative therapists, and psychodynamic therapists focused mostly on the environmental frame. The eclectic therapists mentioned the following environmental subgroups: childhood, trauma, loss of a sense of control, and culture. The integrative therapists mentioned childhood, trau$\mathrm{ma}$, loss of a sense of control, and unspecified environmental factors. The psychodynamic therapists mentioned loss of a sense of control followed by childhood and upbringing.

The biological frame was mentioned equally by the CBT and humanistic therapists. The psychological frame was mentioned mostly by the eclectic therapists. The environmental frame was mentioned the most by the psychodynamic therapists. The combination of environmental and biological frames was mentioned mostly by the humanistic therapists, and the combination of several frames was mentioned mostly by the trauma therapists.

\section{Frames and Clinical Experience}

The third research question explored the association between the psychotherapists' views on the development of disorders and their length of experience as a therapist. In the present sample of psychotherapists, length of experience as a therapist ranged from 3 to 38 years. Frames were analyzed in relation to years of experience as a therapist. Two groups were created based on years of experience. The data showed that 7 therapists had less than 10 years' experience as a therapist and 9 had significantly more than 10 years' experience. Thus, two groups were created: therapists with less than 10 years of experience and therapists with more than 10 years of experience. The number of therapists in the two groups are unequal, and hence the data require normalization before comparisons can be made. The normalized data for frames and years of experience as a therapist are shown in Table 4.

The combination of environmental and biological frames was mentioned slightly more by the therapists with more than 10 years' experience whereas single frames were mentioned slightly more by the therapists with less than 10 years' experience.

The environmental frame was the most frequently mentioned frame in both groups. However, the distributions in the subgroups of environmental factors were different. The less experienced therapists focused more on childhood and upbringing subgroup while more experienced therapists focused equally on the three subgroups of childhood and upbringing, trauma, and loss of a sense of control. Loss of a sense of control was not mentioned by the less experienced therapists whereas psychological factors and cultural factors were not mentioned by the more experienced therapists.
Table 2 Therapeutic Orientations of Psychotherapists

\begin{tabular}{lllllll}
\hline Therapies & $\begin{array}{l}\text { Cognitive- } \\
\text { Behavioral } \\
\text { Therapy }\end{array}$ & $\begin{array}{l}\text { Integrative } \\
\text { Therapy }\end{array}$ & $\begin{array}{l}\text { Trauma } \\
\text { Therapy }\end{array}$ & $\begin{array}{l}\text { Eclectic } \\
\text { Therapy }\end{array}$ & $\begin{array}{l}\text { Psychodynamic } \\
\text { Therapy }\end{array}$ & $\begin{array}{l}\text { Humanistic } \\
\text { Therapy }\end{array}$ \\
\hline Frequency & 7 & 6 & 4 & 4 & 2 & 2 \\
\hline
\end{tabular}


Table 3 Normalized data: Frames according to the therapeutic approaches of psychotherapists and relative frequencies (RF) in percentages

\begin{tabular}{|c|c|c|c|c|c|c|c|}
\hline Frames & $\begin{array}{l}\text { Cognitive Behavioral } \\
\text { Therapy }\end{array}$ & $\begin{array}{l}\text { Eclectic } \\
\text { Therapy }\end{array}$ & $\begin{array}{l}\text { Humanistic } \\
\text { Therapy }\end{array}$ & $\begin{array}{l}\text { Integrative } \\
\text { Therapy }\end{array}$ & $\begin{array}{l}\text { Psychodynamic } \\
\text { Therapy }\end{array}$ & $\begin{array}{l}\text { Trauma } \\
\text { Therapy }\end{array}$ & Totals \\
\hline Single Frames & 13 & 19 & 12 & 17 & 15 & 9 & 85 \\
\hline 1. Biological Factors & $3(7.14 \%)$ & & $3(7.69 \%)$ & $2_{(5.26 \%)}$ & & & 8 \\
\hline 2. Psychological Factors & & $7(18.75 \%)$ & & $1_{(2.63 \%)}$ & & & 8 \\
\hline 3. Environmental Factors & $10_{(25 \%)}$ & $12_{(31.25 \%)}$ & $9_{(23.08 \%)}$ & $14_{(36.84 \%)}$ & $15_{(40 \%)}$ & $9_{(23.53 \%)}$ & 68 \\
\hline a. Childhood and Upbringing & $5_{(14.29 \%)}$ & $5_{(12.50 \%)}$ & $3_{(7.69 \%)}$ & $10_{(26.32 \%)}$ & $4_{(10 \%)}$ & & 27 \\
\hline b. Trauma & & $2(6.25 \%)$ & $3(7.69 \%)$ & $1_{(2.63 \%)}$ & & $9_{(23.53 \%)}$ & 15 \\
\hline c. Loss of Sense of Control & $1_{(3.57 \%)}$ & $2(6.25 \%)$ & $3(7.69 \%)$ & $1_{(2.63 \%)}$ & $11_{(30 \%)}$ & & 19 \\
\hline d. Cultural Factors & & $2_{(6.25 \%)}$ & & & & & 2 \\
\hline $\begin{array}{l}\text { e. Unspecified } \\
\text { Environmental Factors }\end{array}$ & $3(7.14 \%)$ & & & $2_{(5.26 \%)}$ & & & 5 \\
\hline Combinations of Frames & 16 & 7 & 18 & 7 & 8 & 20 & 76 \\
\hline $\begin{array}{l}\text { 1. Combination of } \\
\text { Environmental } \\
\text { and Biological Frames }\end{array}$ & $12(32.14 \%)$ & $5_{(12.50 \%)}$ & $15_{(38.46 \%)}$ & $5(13.16 \%)$ & $4_{(10 \%)}$ & $13_{(35.29 \%)}$ & 54 \\
\hline 2. Combination of All Frames & $4_{(10.71 \%)}$ & $2(6.25 \%)$ & 3 (7.69\%) & $2_{(5.26 \%)}$ & $4_{(10 \%)}$ & $7(17.65 \%)$ & 22 \\
\hline Totals & $38_{(100 \%)}$ & $38_{(100 \%)}$ & $38_{(100 \%)}$ & $38_{(100 \%)}$ & $38_{(100 \%)}$ & $38_{(100 \%)}$ & 228 \\
\hline
\end{tabular}

\section{Discussion}

This study offers an insight into therapists' opinions and understandings on the development of psychological disorders. Specifically, psychotherapists' views were studied by applying Goffman's frame theory to interview data. Factors underlying the emergence of psychological disorders mentioned by the participating therapists were grouped into frames. Two distinct, overarching categories of frames were identified: single frames and combinations of frames. Factors constituting single frames were mentioned and discussed in isolation, while in combinations of frames factors from two or more frames were viewed as contributing to the development of disorders. The single frames category comprised the sub-frames of biological, psychological, and environmental factors and the combinations of frames category comprised two subcategories: the combination of factors in the environmental and biological frames, and the combination of factors from several frames.

These frames were then examined in relation to the participants' therapeutic orientation and years of clinical experience. The main therapeutic orientations reported by the psychotherapists were psychodynamic, cognitive behavioral, humanistic, integrative, and eclectic. The factors most frequently mentioned across all the present therapists' orientations were those subsumed under the over-arching environmental frame, i.e., childhood and upbringing, trauma, and loss of a sense of
Table 4 Normalized data: Frames and Years of Experience as a Psychotherapist

\begin{tabular}{lll}
\hline Frames & $\begin{array}{l}\text { Less than } 10 \\
\text { years of experience }\end{array}$ & $\begin{array}{l}\text { More than } 10 \\
\text { years of experience }\end{array}$ \\
\hline Single Frames & $\mathbf{2 1}$ & $\mathbf{1 7}$ \\
1.Biological Factors & 1 & 1 \\
2.Psychological Factors & 5 & 0 \\
3.Environmental Factors & 15 & 16 \\
a. Childhood and Upbringing & 11 & 5 \\
b. Trauma & 1 & 5 \\
c. Loss of Sense of Control & 0 & 5 \\
d. Cultural Factors & 1 & 0 \\
e. Unspecified Environmental Factors & 1 & 1 \\
Combinations of Frames & $\mathbf{1 1}$ & $\mathbf{1 4}$ \\
1.Combination of Environmental and Biological Frames & 7 & 10 \\
2.Combination of Several Frames & 4 & 4 \\
Totals & $\mathbf{4 7}$ & $\mathbf{4 7}$ \\
\hline
\end{tabular}


control, followed by the combination of environmental and biological frames. With respect to length of clinical experience, the therapists who had practiced for more ten years discussed combinations of factors slightly more often than single factors. Of the therapists who only mentioned factors within the environmental frame, those with less than ten years of experience mostly discussed the single factor of childhood and upbringing, whereas those with more than ten years' experience assigned equal importance to childhood and upbringing, trauma, and loss of a sense of control.

Previous studies have shown that therapists differentiate between the contribution of neurobiological and psychological factors to the development of disorders (Miresco and Kirmayer, 2006). Therapists also tend to focus on either biological or on psychological and environmental factors (Ahn et al., 2009). In this study, however, the therapists more often discussed biological factors in combination with environmental factors. The environmental frame, which was in the single frames category, was the frame most often mentioned by the present participants. Although environmental factors were perceived as a single factor, they functioned within a larger scheme of psychological processes. Environmental factors such as childhood and upbringing were discussed in relation to negative cognitions and a negative perception of self and the world. Childhood and upbringing, including negative childhood events, neglect, and abuse, were also viewed as linked to trauma. Trauma was also linked to neurological processes as well as to the development of negative cognitions and schemas.

In addition, the frame comprising the combination of factors from the environmental and biological frames was the second most often mentioned frame. Both perspectives confirm the importance of the biomedical model in the emergence of mental disorders. However, some of the therapists in the present study believed that genetic or biological factors need an environmental trigger to induce a mental disorder, while others believed that genetics or biological factors alone can lead to a disorder. These results accord with previous findings of disagreement among therapists on a clear-cut etiological basis for mental disorders (Ahn et al., 2006).

Whereas the literature on therapeutic approaches shows a clear distinction between each approach, this was not the case with the therapists in this study. In the literature, psychodynamic therapy focuses on unconscious desires and childhood events as a basis for mental disorders (Cave, 1999). In this study, the therapists with a psychodynamic approach focused on loss of a sense of control more than on childhood and upbringing. The cognitive-behavioral approach is known for positing learning processes and cognitions as a basis for mental disorders, while in the humanistic approach deviation of the self is considered the root cause (Cave, 1999). In this study, however, both the cognitive behavioral and the humanistic therapists mostly combined biological and environmental factors. While eclectic therapists generally choose therapy techniques based on effectiveness, the present eclectic therapists focused mostly on environmental and psychological factors. Integrative therapists, in turn, combine similar techniques from different therapeutic approaches, yet in this study they focused on childhood and upbringing.

Previous research has also shown that length of experience alters the way therapists view mental disorders and increases their tolerance of ambiguity (Dawson, 2018). The present results concur with this notion, showing that therapists with more than ten years of experience explained the development of disorders using a combination of factors slightly more often than those with less experience.

The findings of this study could be viewed as evidence of an increased attempt by therapists at integrating multiple factors when explaining the emergence of mental disorders. The psychotherapists combined biological and environmental factors rather than viewing these categories as independent of each other. Within the environmental frame, different subgroups of factors were also connected, showing that the therapists viewed the factors underlying the development of psychological disorders as complex and intertwined.

In the subgroups of environmental factors, trauma and loss of a sense of control had features in common. They were both discussed as an outcome of life events in general or of events over which individuals perceive they have no power, and thus could be linked to an impaired sense of agency. War and warrelated crises were also mentioned as environmental factors leading to the emergence of disorders. Given that the therapists in this study are Lebanese, this finding is unsurprising, as war has been a recurrent situation for decades in Lebanon. Another finding was the mention by some therapists of resilience as a protective factor. Despite the many factors that could lead to mental disorders, the availability of support in an individual's life was seen as an aid in developing resilience. This further indicates the complex nature of psychological disorders and highlights the impact of protective factors on their development.

Examination of the frames in relation to the therapists' different therapeutic orientations and understandings of the development of disorders revealed several common features. The therapists acknowledged and combined several concepts in their analysis of psychological disorders, showing that they understood the emergence of disorders from multiple standpoints. They incorporated several therapeutic orientations and did not show excessive commitment to any one perspective. For example, the trauma therapists were expected to focus mostly on trauma and its effects on the development of disorders; however, in this study they focused on combinations of factors, including trauma and biology.

These findings may be explained by the fact that some therapists utilized two or more therapeutic approaches in their practice. Another possible explanation is that therapists no longer choose a single therapeutic approach and adhere to it exclusively. Moreover, the undeniable importance of the biomedical 
model in psychiatry could also be an explanation for the combination of therapeutic approaches. The measurable nature of the biomedical model, including neuropsychology, adds credibility to psychotherapy. Another explanation could be the increasing research on the effectiveness of different psychological therapies, thereby revealing the complexity of human psychology and the rejection of a one-size-fits-all concept.

In this study several therapists referred to applying trauma therapy and EMDR. This finding could be explained by the fact that this study was conducted in a country that recurrently struggles with war. It may also indicate that trauma therapy is gaining prevalence and a possible legitimate therapeutic position.

Therapists with more years of experience as a therapist felt more at ease in combining different therapeutic approaches as well as in developing their own approaches to understanding human psychology. These findings could indicate that more experienced therapists are more comfortable with the complexities of mental disorders, and hence perceive multiple rather than single factors as important (Ronnestad and Skovholt, 2012). The more experienced therapists are, the more willing they are to consider and combine underlying developmental factors with triggering factors in their efforts to understand psychological disorders. Therapists with less experience might find this process overwhelming and instead prefer to focus on single factors. Moreover, for new therapists, compartmentalizing the development of disorders possibly simplifies the clinical situation and provides a reassuring sense of control in treating clients.

\section{Strengths and Limitations}

Participants' rights and privacy were protected throughout the study. In addition, transparency was observed, and participants were given a detailed description of the research process. Participants consented to the use of their data in publishing the results. Participant anonymity was an important issue: names and personal information were removed from all transcripts before they were saved. Similarly, audio recordings were saved and stored on private files and contained no personal details.

The study findings may be of interest to psychotherapists and individuals interested in psychology, especially since they indicate how psychotherapists understand the development of mental disorders. This study also provides an idea about the influence of psychotherapists' therapeutic orientation and length of clinical practice on how they perceive the emergence of mental disorders. The study may also encourage cross-country comparative research on development and change in psychotherapy.

The findings of this study may be influenced by the mention of loss of a sense of control when informing participants about the purpose of the study. However, only one therapist discussed this issue in the interview data. The selection of psychotherapists may have also biased the results, as all the participating therapists work in Beirut, the Lebanese capital. The therapists' views and their interpretation of the interview questions, although general, could have been affected by the types of mental disorders they treat in their clinics. Moreover, most of the participants worked in private clinics, which do not commonly treat clients with severe mental health conditions.

Lebanon is a trilingual country, and it is common for therapists to have studied psychology in English. Some psychotherapists teach psychology in universities and provide psychotherapy for clients in English. Some of the participants had degrees from English-speaking countries and others had received psychotherapy training provided internationally in English. The therapists did not seem to have difficulties in explaining their opinions and using psychological terminology in English. Moreover, only one interview, conducted in Arabic, was translated into English. For these reasons, the use of English in the interviews is not seen as a limitation.

\section{Conclusion}

This study offers a unique perspective on how psychotherapists understand the development of psychological disorders. The results showed that therapists were flexible and did not rely on any single approach but instead combined different psychological theories. The longer the therapists had practiced, the more complex their views on the emergence of mental disorders. Trauma therapy was often mentioned, and several therapists reported applying trauma therapy in their clinics. Future research could examine the prevalence of trauma therapy and EMDR therapy in other countries.

This study also provides valuable data from a small country that faces crises caused by war. Since war is an ongoing reality in many countries globally, it would be interesting to know whether therapists in different countries would also cite war and war-related crises as environmental factors when pondering the development of disorders. Future research could consider how psychotherapists explain environmental factors based on the issues prevailing in their communities.

\section{Appendix 1}

\section{Interview Questions}

1- Which of the following best describes your therapeutic approach?

$\square$ Psychoanalytic $\square$ Behavioral $\square$ Cognitive $\square$ Humanistic $\square$ Systemic $\square$ Narrative $\square$ Solution-Focused $\square$ Collaborative

$\square$ Integrative 
2- What degrees you have completed? And in which fields?

3- How many years have you worked as a psychotherapist?

4- In which language did you study psychology?

5- Do you feel comfortable explaining your opinions clearly in English?

6- Clarify, based on your approach, how psychological disorders develop.

7- How would you define a triggering factor?

8- What are the most recurrent triggering factors for psychological disorders that you have observed? Please give examples.

9- From your experience, do you think there is a common trigger for most psychological disorders? If so, please explain.

10- How would you define loss of sense of control?

11- In your opinion, does loss of sense of control play a part in the development of psychological disorders? If yes, how?

12- If available, please describe any cases in which loss of sense of control triggered the onset of a psychological disorder.

13- For further research, do you believe a causal relationship between loss of sense of control and the onset of psychological disorders should be studied?

Code Availability Not applicable.

Author Contributions All authors made a substantial contribution to the concept of the article, the interpretation of data, and the revision of article drafts. The collection of the data and analysis was done by the corresponding author.

Funding Open access funding provided by University of Jyväskylä (JYU).

Data Availability Consent for sharing the data was not provided by participants.

\section{Declarations}

Consent to Participate Informed consent, both verbal and written, were obtained from all participants included in this study.

Consent to Publish Participants consented to the use of the data for publishing.

Conflicts of Interest The authors declare that there is no conflict of interest.

Ethical Approval All procedures performed in studies involving human participants were in accordance with the ethical standards of the institutional and/or national research committee and with the 1964 Helsinki declaration and its later amendments or comparable ethical standards.
Open Access This article is licensed under a Creative Commons Attribution 4.0 International License, which permits use, sharing, adaptation, distribution and reproduction in any medium or format, as long as you give appropriate credit to the original author(s) and the source, provide a link to the Creative Commons licence, and indicate if changes were made. The images or other third party material in this article are included in the article's Creative Commons licence, unless indicated otherwise in a credit line to the material. If material is not included in the article's Creative Commons licence and your intended use is not permitted by statutory regulation or exceeds the permitted use, you will need to obtain permission directly from the copyright holder. To view a copy of this licence, visit http://creativecommons.org/licenses/by/4.0/.

\section{References}

Ahn, W. K., Flanagan, E. H., Marsh, J. K., \& Sanislow, C. A. (2006). Beliefs about essences and the reality of mental disorders. Psychological Science, 17(9), 759-766. https://doi.org/10.1111/j. 1467-9280.2006.01779.x

Ahn, W., Proctor, C. C., \& Flanagan, E. H. (2009). Mental health clinicians' beliefs about the biological, psychological, and environmental bases of mental disorders. Cognitive Science, 33(2), 147-182. https://doi.org/10.1111/j.1551-6709.2009.01008.x

American Psychiatric Association. (2013). Diagnostic and statistical manual of mental disorders (5th ed.). https://doi.org/10.1176/appi. books. 9780890425596

Cave, S. (1999). Therapeutic Approaches in Psychology (1st ed.). Routledge. https://doi.org/10.4324/9780203136270

Dawson, G. C. (2018). Years of clinical experience and therapist professional development: A literature review. Journal of Contemporary Psychotherapy: On the Cutting Edge of Modern Developments in Psychotherapy, 48(2), 89-97. https://doi.org/10.1007/s10879-0179373-8

Goffman, E. (1974). Frame analysis: An essay on the organization of experience. Harvard University Press.

Leitan, N. D., \& Murray, G. (2014). The mind-body relationship in psychotherapy: Grounded cognition as an explanatory framework. Frontiers in Psychology, 5, 472. https://doi.org/10.3389/fpsyg. 2014.00472

Miresco, M. J., \& Kirmayer, L. J. (2006). The persistence of mind-brain dualism in psychiatric reasoning about clinical scenarios. The American Journal of Psychiatry, 163(5), 913-918. https://doi.org/ 10.1176/ajp.2006.163.5.913

Norcross, J. C., \& Goldfried, M. R. (Eds.). (2005). Handbook of psychotherapy integration (2nd ed.). Oxford University Press. https://doi. org/10.1093/med:psych/9780195165791.001.0001

Ronnestad, M. H., \& Skovholt, T. (2012). The developing practitioner: Growth and stagnation of therapists and counselors (1st ed.). Routledge. https://doi.org/10.4324/9780203841402

Shapiro, D.H. (1994). Manual for the Shapiro control inventory. Behaviordata, Enc. http://controlresearch.net/support-files/SCI manual.pdf

Shaw, E. (2013). Frame analysis. Encyclopedia Britannica. https://www. britannica.com/topic/frame-analysis

Skovholt, T. M. (2012). Becoming a therapist: On the path to mastery. John Wiley and Sons.

Publisher's Note Springer Nature remains neutral with regard to jurisdictional claims in published maps and institutional affiliations. 